\title{
Connective Tissue Disease in a Malawian Man
}

\section{Allain Theresa ${ }^{1}$, Banda Ndaziona ${ }^{1}$, van Gaalen Floris ${ }^{2}$}

1. Dept of Med. College of Medicine, University of Malawi

2.Leiden University Medical Centre

\section{Introduction}

We describe a case of systemic connective tissue disease (CTD) in a Malawian man in whom an autoimmune etiology was likely. Autoimmune CTDs are rarely reported in Africans[2] and there have been no case reports from Malawi. We were fortunate in that we were able to obtain additional tests on this patient including his autoantibody profile and HLA type which helped characterise his problem.

\section{Case Presentantion}

A 17 year-old student was referred to Queen Elizabeth Central Hospital $(\mathrm{QECH})$ for management of progressive breathlessness. At the referring hospital he had been noted to have an abnormal chest X-ray (CXR) and had been treated for smear negative pulmonary TB (pTB). Having completed TB treatment there was no improvement and he was being considered for re-treatment for smear negative TB at the time of referral. At this point his case was reviewed.

Breathlessness, which was worse on exertion, had been present for 18 months. It was not associated with cough or fevers. Joint pain, mainly affecting his hands, developed one month after the breathlessness. It was worse in the morning and associated with stiffness lasting more than 1 hour daily. He had noticed deformity of his hands. He occasionally had dry mouth but denied eye symptoms, Raynauds phenomenon or dysphagia. There was no relevant environmental exposure, past medical or family history. The only previous medication was anti-tuberculous drugs plus paracetamol. He did not drink alcohol nor smoke.

On examination he was of small stature. He had no rashes. His eyes and mouth were normal. There was no cyanosis or lymphadenopathy and blood pressure was normal. The appearance of his hands is shown in figure 1. The skin appeared thickened, shiny and tight with camptodactylia (sclerodactylia). There was no cutaneous calcinosis, ulceration or telangectasia. The distal phalanges were shortened with clubbing and/or pseudoclubbing of the nails. He had swelling and deformity of the wrists but other large joints appeared normal. Chest expansion was reduced bilaterally and auscultation was normal. His spleen was palpable $4 \mathrm{~cm}$ below the costal margin.

$\mathrm{X}$ rays of the hands are shown in Figure 2. The most striking abnormality was acro-osteolysis ${ }^{3}$.

Acro-osteolysis describes a destructive process involving one or more terminal phalnges. It is seen in a number of systemic and local conditions, most commonly systemic sclerosis (SSc) (box 1).

His CXR (figure 3) showed possible hilar adenopathy and bilateral reticulonodular shadowing.

Spirometry confirmed a severe restrictive lung defect; FEV1 1.27, FVC 1.27 (both $<40 \%$ predicted).

Urinanalysis: showed proteinuria + , no haematuria. His serum creatinine was 38 micromol/1 (ref. 62-106). Although the resources of our hospital are limited, through collaboration with colleaguesoverseas we were able to define the patient's immune and biochemical status in more detail.
Figure 1-Photograph of both hands taken when first seen (18 months after symptom onset).

Sclerodactylia and camptodactylia of all fingers except the thumbs can be seen. There is shortening

of the distal phalanges and clubbing of the nails.

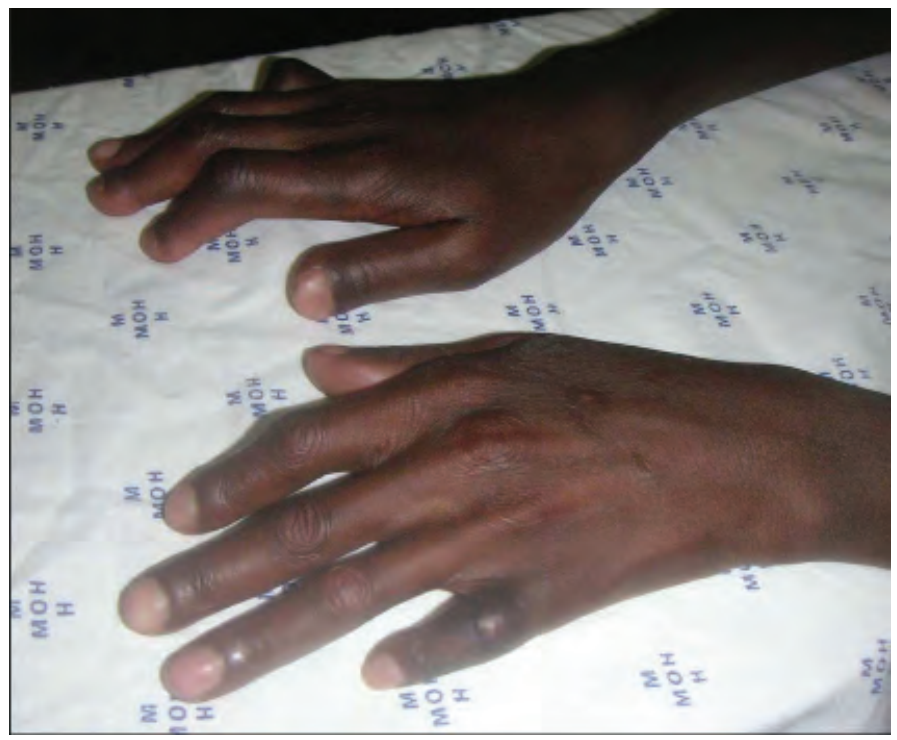

Figure 2 (right and left)- X rays of the hands taken when first seen (18 months after symptom onset).

The MCP joints appear normal. The PIP joints, particularly in digits II and III on the right hand and digit II and IV on the left, show cartilage loss. The DIP joints are difficult to see but all distal phalanges are shortened suggesting acro-osteolysis. It can also be noted that the epiphyseal plate can still be seen and may suggest that he is younger than 17, possibly 13 - 14 years

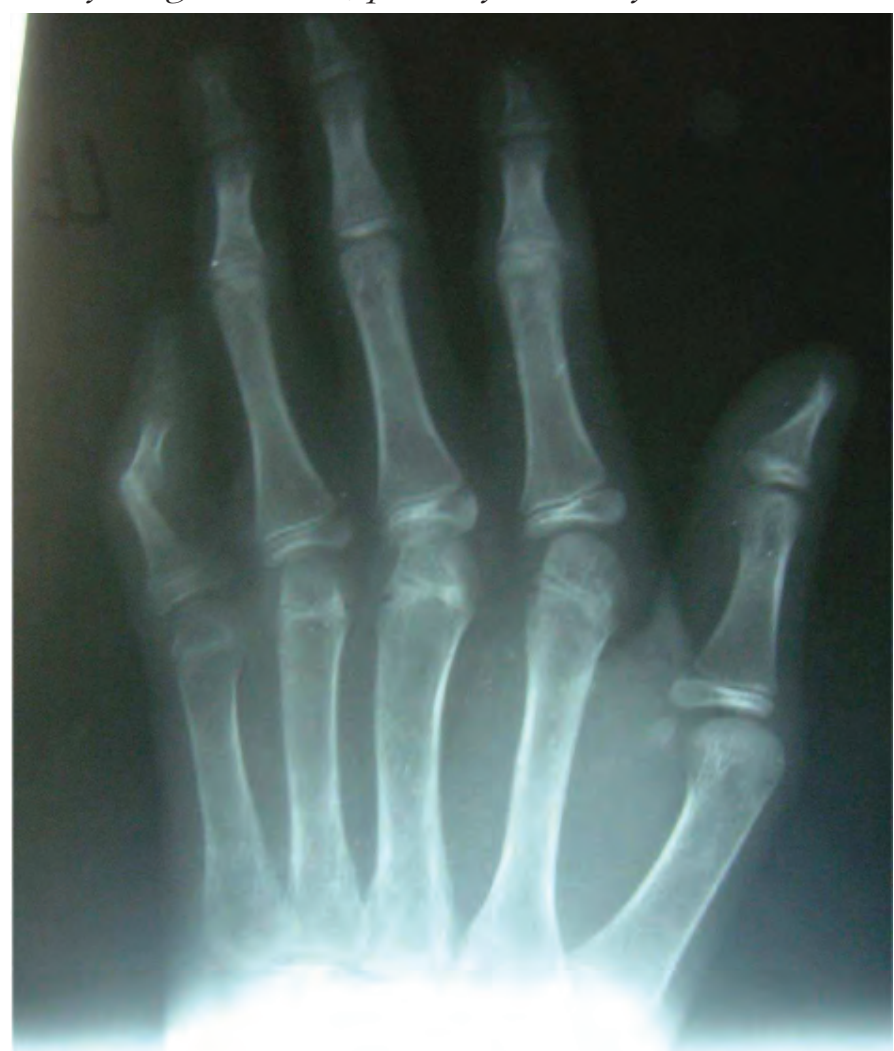




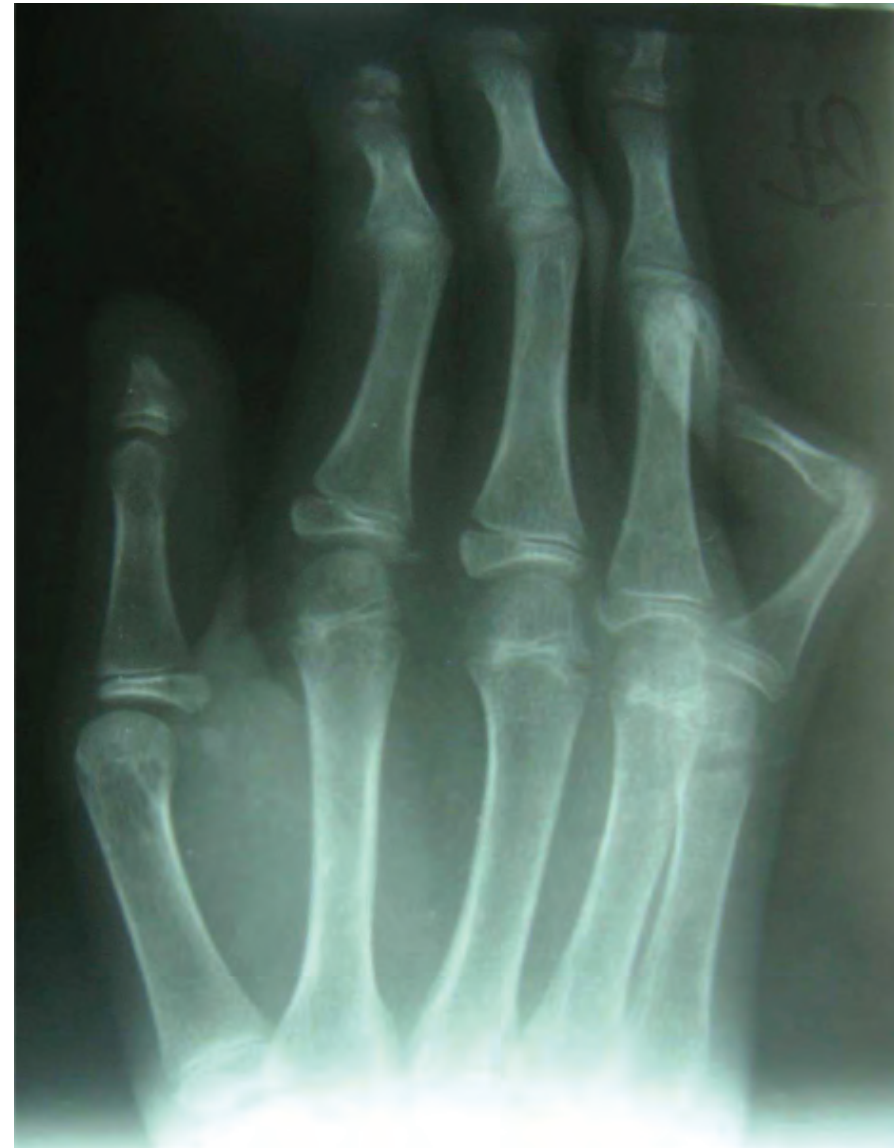

His autoantibody profile was as follows: ANF negative, AntiENA positive, Anti-SSA (anti Ro) strong positive, Anti-SSB (anti La) negative, Anti-RNP negative, Ant-SM negative negative, Anti-Scl 70 (antitopoisomerase 1) negative, AntiJO-1 negative.

Other biochemistry: calcium 2.22mmol/1 (ref. 2.15-2.55), phosphate $1.4 \mathrm{mmol} / 1$ (ref. 0.9-1.5), albumin $40 \mathrm{~g} / 1,25(\mathrm{OH})$ vitamin D 62nmol/1 (ref. 62-120), CRP $<15$.

HLA Class 1 typing by PCR-SSP: Alleles (serological equivalents in brackets) : A*02 (A2), A*74 (A74), B*15 (B72), B*45 (B45), $\mathrm{Cw}^{*} 02$ (Cw2), Cw*16 (Cw16), HLA class 11 typing by PCR-SSP: Alleles: DRB1*11 (DR11), DRB1*13 (DR13), DRB $3 * 01 / 02$ (DR52), $\quad \mathrm{DRB} 4 *=, \quad \mathrm{DRB} 5 *=, \quad \mathrm{DQB} 1 * 03$ (DQ7), DQB1*06 (DQ6).

He was treated with NSAIDS, counseling and physiotherapy with symptomatic improvement. He reattended 18 months later due to deteriorating breathlessness. He was now short of breath at rest and had swollen ankles. Examination revealed progression of the skin changes in his hands with

increased skin thickening, increased flexion and small ulcers overlying the PIP joints. He was cyanosed, O2 saturation $77 \%$, blood pressure $90 / 60 \mathrm{mmHg}$ and had signs of right ventricular failure. CXR showed widening of the cardiac silhouette, lung fields similar to previously. Spirometry was essentially unchanged. Echocardiography revealed massive dilation of the right ventricle and right atrium. A diagnosis of cor-pulmonale was made. He did not respond to treatment with diuretics and died peacefully at home 2 months later, under the care of the palliative care team.

\section{Discussion}

The patients prominent clinical features were pulmonary fibrosis and arthropathy. He had sclerodactylia with pseudoclubbing $^{4,5}$ due to acro-osteolysis and/or clubbing due to
(18 months after symptom onset).

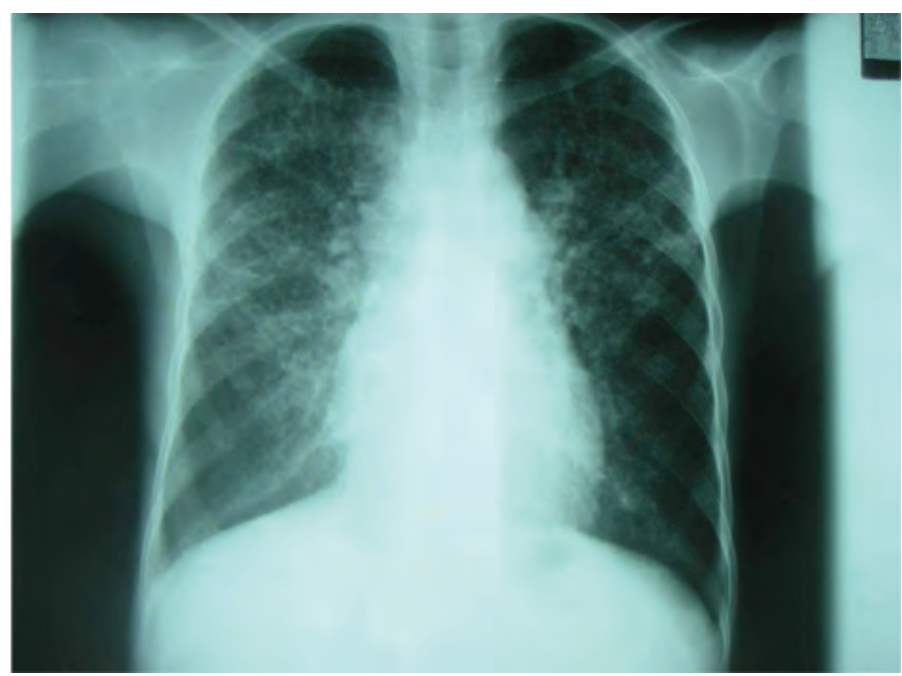

pulmonary disease with joint space narrowing in the proximal inter-phalyngeal (PIP) joints. These clinical features most closely fit with systemic sclerosis, although there were some a typical features. The radiograph findings are compatible with changes seen in scleroderma but not with rheumatoid arthritis (RA).

Systemic sclerosis is said to occur world-wide, although the only reports from Sub-Saharan Africa are from South Af$\mathrm{rica}^{2}$. The incidence is $10 /$ million population/year. The male :female ratio is 3:1 and the peak age of onset 30-50 years. Scleroderma-like syndromes can be triggered by environmental exposure to vinyl chloride, silica dust, adulterated rape seed oil and trichloroethylene but exposure to these was not reported in our patient. Acro-osteolysis can be seen in a number of conditions ${ }^{3}$ box ${ }^{1}$, but in this case SSc was most likely. In patients with SSc, acro-osteolysis is significantly associated with digital ulceration and systemic complications, such as pulmonary fibrosis ${ }^{1}$. This combination of features was seen in our patient. The overall prognosis for SSc is $55 \%$ 10 year survival, with most patients dying from pulmonary, cardiac or renal involvement.

As in our case, $80 \%$ of SSc patients have positive anti-nuclear antibodies, however the pattern of autoantibodies in our patient is different from that typically described. Disseminated $\mathrm{SSc}$ is associated with Scl-70/antitopoisomerase 1 in up to $50 \%$ of cases. In this patient the ENA was positive, with a strong SSA signal. SSA or anti-Ro antibodies are primarily found in patients with SLE and Sjögren's syndrome. Clinically our patient did not have either of these conditions. SSA antibodies are infrequently seen in other connective tissue diseases such as scleroderma, polymyositis, mixed connective tissue disease, and RA. Among patients with anti-Ro/ SSA antibodies and initially undifferentiated connective tissue disease, only around 25\% evolve to a more defined CTD (most often Sjögren's).

Racial differences in the clinical features, auto-antibody profile and genetic associations of SSc have been described. Case series reported from the USA and South Africa demonstrate that among patients with systemic sclerosis blacks are more likely to have progressive pulmonary interstitial fibrosis and worse outcomes ${ }^{2,6,7}$. In caucasian populations SSc is associated with HLA A1, B8, DR3, DR5, DRw52 and DR4 which were not present in our patient. However, our patient had HLA class 11 type DRB1*11. This HLA type has been 
described in association with Scl-70 in African Americans ${ }^{6}$ and in association with pulmonary fibrosis in black South Africans with $\mathrm{SSc}^{8}$.

\section{Conclusion}

In summary this is a case of a young patient with a rapidly progressive disease with several features suggesting an autoimmune process, particularly systemic sclerosis. However, he also had severe erosive arthopathy and an autoantibody profile suggesting Sjogren's syndrome consistent with the observation that CTDs in Africans may present differently than in Caucasians and have different underlying immune mechanisms. The case illustrates an important lesson; that some patients with a common presentation (breathlessness and an abnormal CXR), may have rare conditions. In our patient a thorough history and examination revealed additional findings that pointed to the correct

diagnosis.

\section{References}

1. Avouac, J., et al., Radiological hand involvement in systemic sclerosis. Ann Rheum Dis, 2006. 65: p. 1088-92.

2. Tager, R.E. and M. Tikly, Clinical and laboratory manifestations of systemic sclerosis (scleroderma) in Black South Africans. Rheumatology, 1999. 38: p. 397-400.

3. Kemp, S.S., M.K. Dalinka, and H.R. Schumacher, Acro-osteolysis. Etiologic and radiological considerations. JAMA, 1986. 255(15): p. 2058-61.

4. Monaco, A.L., M. Govoni, and F. Trotta, Digital clubbing or digital pseudoclubbing in Systemic Sclerosis. JCR, 2006. 12(2): p. 97.

5. Farzaneh-Far, A., Pseudoclubbing. New Engl J med, 2006. 354(15): p. e14.

6. Kuwana, M., et al., Influence of ethnic background on clinical and serological features in patients with systemic sclerosis and anti-DNA topoisomerase 1antibody. Arthritis Rheum, 1999. 42: p. 465-74.

7. Reveille, J.D., et al., Systemic sclerosis in 3 US ethnic groups: a comparison of clinical, sociodemographic, serologic, and immunogenetic determinants. Semin Arthritis Rheum, 2001. 30: p. 332-46.

8. Tikly, M., et al., HLA and ethnic associations among systemic sclerosis patients with anticentromere antibodies. Tissue Antigens, 2004. 63: p. 487-90.

We would like to thank the Department of Rheumatology, Leiden University Medical Centre, The Netherlands for providing the auto antibody profile and additional biochemistry and Dr Mas Chaponda and colleagues at the Department of Transplant Immunology at the Royal Liverpool University Hospital, UK for the HLA typing.

\section{(KENTAM PRoducts Limited FORALLYOUR PHARMACEUTICAL, LAB AND
MEDICAL INSTRUMENT REQUIREMENTS
SERVING CLINICS, PHARMACIES \& HOSPITALS:}

- Extensive quality product listing at competitive prices - Our manufactured brands

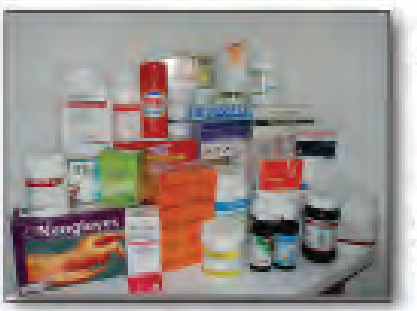

Analgesics/Antipyretics/Antiarthritics.Antibacterial/Antiblotic. AnticoldVExpectorants. Antidiabetics. Antidiarrhoel.Antifungal. Antihistamine/antiallergen.Antimalarials.Antimicrobial oral. Antimicrobial topical. Antithelmintics' Antiortozoals, Antitubercualr. Artiuloerants/Antiennetics, Beta-Lactum. Blood \& Heamotopoetics Canliac Care. Cephalosporins. First Aid. IV Infisions. Laboratory agents and Instruments. Medicals Instruments. Ophthalmic Preparations. Psychotropic. Steroids. Vitamins/Nutritional Supplenents.

* Onsite purchasing; timely and reliable monthly visits and deliveries nationwide

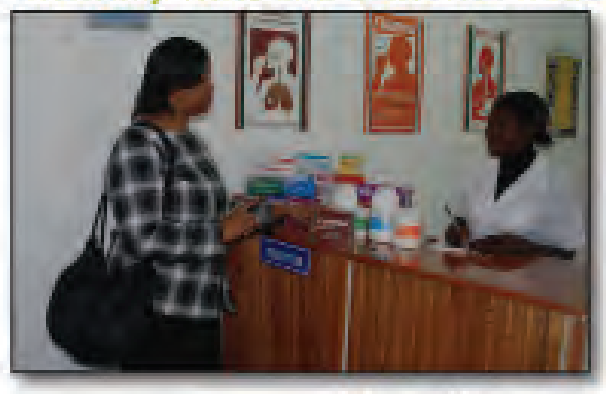

\section{- Pharmacy, Medicines \& Poisons Board Licensed for both Manufacturing: E Wholesale Distribution}

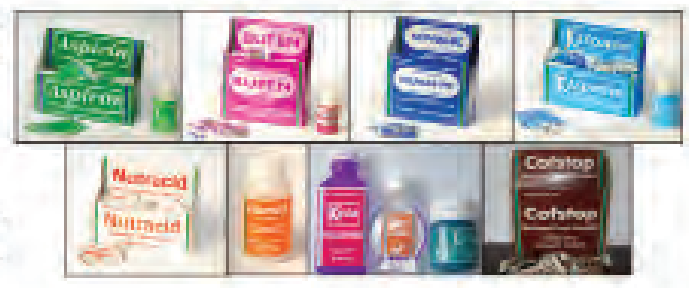

+ Friendly \& knowledgeable staff that prioritises the client's needs - alwayst

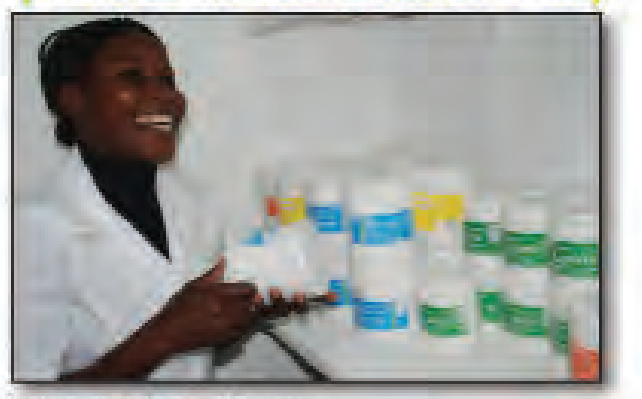

For orders, contact: Kentam Products Ltd., Luwinga Industrial Area, Mzuzu Tel:01-320-588/643 Fax: 01-320-756 Email: kentam@africa-online.net or cmtegha@kentam.co.mw 